\title{
CXCL1, CCL2, and CCL5 modulation by microbial and biomechanical signals in periodontal cells and tissues - in vitro and in vivo studies
}

\author{
Birgit Rath-Deschner ${ }^{1} \cdot$ Svenja Memmert ${ }^{1,2} \cdot$ Anna Damanaki $^{3} \cdot$ Marjan Nokhbehsaim $^{2} \cdot$ Sigrun Eick $^{4} \cdot$ Joni A. Cirelli $^{5}$. \\ Werner Götz ${ }^{1}$. James Deschner ${ }^{3} \cdot$ Andreas Jäger $^{1} \cdot$ Andressa V. B. Nogueira $^{3}$
}

Received: 24 October 2019 / Accepted: 12 February 2020 / Published online: 2 March 2020

(C) The Author(s) 2020

\begin{abstract}
Objectives This study was established to investigate whether the chemokines CXCL1, CCL2, and CCL5 are produced in periodontal cells and tissues and, if so, whether their levels are regulated by microbial and/or mechanical signals.

Materials and methods The chemokine expression and protein levels in gingival biopsies from patients with and without periodontitis were analyzed by RT-PCR and immunohistochemistry. The chemokines were also analyzed in gingival biopsies from rats subjected to experimental periodontitis and/or orthodontic tooth movement. Additionally, chemokine levels were determined in periodontal fibroblasts exposed to the periodontopathogen Fusobacterium nucleatum and mechanical forces by RT-PCR and ELISA.

Results Higher CXCL1, CCL2, and CCL5 levels were found in human and rat gingiva from sites of periodontitis as compared with periodontally healthy sites. In the rat experimental periodontitis model, the bacteria-induced upregulation of these chemokines was significantly counteracted by orthodontic forces. In vitro, F. nucleatum caused a significant upregulation of all chemokines at 1 day. When the cells were subjected simultaneously to $F$. nucleatum and mechanical forces, the upregulation of chemokines was significantly inhibited. The transcriptional findings were paralleled at protein level.

Conclusions This study provides original evidence in vitro and in vivo that the chemokines CXCL1, CCL2, and CCL5 are regulated by both microbial and mechanical signals in periodontal cells and tissues. Furthermore, our study revealed that biomechanical forces can counteract the stimulatory actions of $F$. nucleatum on these chemokines.

Clinical relevance Mechanical loading might aggravate periodontal infection by compromising the recruitment of immunoinflammatory cells.
\end{abstract}

Keywords Orthodontic tooth movement $\cdot$ Fusobacterium nucleatum $\cdot$ Periodontitis $\cdot$ Gingivitis $\cdot$ Periodontium

Birgit Rath-Deschner

brathde@uni-bonn.de

1 Department of Orthodontics, Center of Dento-Maxillo-Facial Medicine, University of Bonn, Welschnonnenstrasse 17, 53111 Bonn, Germany

2 Section of Experimental Dento-Maxillo-Facial Medicine, Center of Dento-Maxillo-Facial Medicine, University of Bonn, Bonn, Germany

3 Department of Periodontology and Operative Dentistry, University Medical Center of the Johannes Gutenberg University, Mainz, Germany

4 Department of Periodontology, Laboratory for Oral Microbiology, University of Bern, Bern, Switzerland

5 Department of Diagnosis and Surgery, School of Dentistry at Araraquara, Sao Paulo State University, UNESP, Araraquara, Brazil

\section{Introduction}

Periodontitis is a chronic inflammatory disease characterized by the loss of alveolar bone and clinical attachment $[1,2]$. If untreated, the disease can finally result in tooth loss and therefore compromised masticatory performance. Although the disease is multifactorial, microorganisms located on the tooth surface and next to the periodontal tissues are a prerequisite for the initiation and development of periodontitis. Among these microorganisms, Fusobacterium nucleatum plays a critical role, because it acts as a bridge bacterium, i.e., it enables other pathogenic microorganisms to colonize the biofilm [3, 4]. These microorganisms in concert with other risk factors of periodontitis induce an inflammatory host response which is mounted to control and finish the microbial attack. The response by the host comprises a lot of professional and 
accessory immunoinflammatory cells, which produce a wide array of inflammatory mediators, matrix-degrading enzymes, and osteoclast-activating molecules [2].

Chemokines, i.e., chemotactic cytokines, are of utmost importance in inflammatory processes, as they ensure a sufficient recruitment of immunoinflammatory cells to the site of microbial infection, e.g., gingiva [5]. Therefore, they play a critical role in chronic inflammatory diseases such as atherosclerosis, osteoarthritis, rheumatoid arthritis, obesity, and periodontitis [6-11]. CXCL1, also referred to as GRO $\alpha$, is a strong chemoattractant for neutrophils which form the first line of defense in the gingival sulcus. The neutrophils can eliminate or reduce bacteria through several mechanisms, such as phagocytosis, extracellular killing by secretion of granule, and formation of extracellular traps $[12,13]$. In contrast to CXCL1, the chemokines CCL2 and CCL5, also known as MCP-1 and RANTES, respectively, mainly facilitate the recruitment of monocytes, macrophages, and $\mathrm{T}$ lymphocytes, which are located below the gingival epithelium, where they form the second line of defense [13]. Therefore, regulation of the CXCL1, CCL2, and CCL5 production by microorganisms or other stimuli/stressors will have an enormous impact on periodontal inflammation and tissue homeostasis.

During mastication but especially in orthodontic tooth movement, teeth and, therefore, the surrounding periodontal tissues are subjected to biomechanical loading. Biomechanical stimuli are critical for the maintenance, formation, and remodeling of soft and hard tissues, but it has also been demonstrated that overloading can lead to inflammation, proteolysis, and bone resorption $[14,15]$. Therefore, like in periodontitis, chemokine levels are altered in periodontal tissues under biomechanical loading conditions. The potential of biomechanical forces to regulate inflammation and tissue formation is clinically used in orthodontic therapy, which aims to correct malposition of teeth.

So far, little is known whether and with what consequence microbial and biomechanical signals interact on periodontal cells and tissues. Therefore, this study was established to investigate whether the chemokines CXCL1, CCL2, and CCL5 are produced in periodontal cells and tissues and, if so, whether their levels are regulated by microbial and/or biomechanical signals in vitro and in vivo.

\section{Materials and methods}

\section{Human gingival biopsies from periodontally healthy and periodontitis sites}

The healthy gingival biopsies and the inflamed gingival tissues were obtained from different patients. Healthy gingiva $(n=6)$ became available during wisdom tooth surgery and tooth extraction procedures for orthodontic indications in the
Department of Oral Surgery, University of Bonn. Inflamed gingival tissues were obtained from periodontitis patients $(n=6)$ during tooth extraction procedures. These teeth had to be removed for periodontal reasons. Approval of the Ethics Committee of the University of Bonn was obtained (\#043/11), and written informed consent was provided by the patients. Exclusion criteria were presence of systemic diseases and/or smoking habits. Gingival sites with a gingival index $=0$ (no clinical inflammation), periodontal probing depth $\leq 3 \mathrm{~mm}$, no clinical attachment loss, and no radiographic bone loss were considered periodontally healthy, whereas gingival sites with a gingival index $>1$, periodontal pocket depth $\geq 5 \mathrm{~mm}$, clinical attachment loss $\geq 3 \mathrm{~mm}$, and radiographic bone loss were considered periodontally diseased (periodontitis).

\section{Gingival biopsies from rats subjected to experimental periodontitis and/or orthodontic tooth movement}

The animal experiments were performed according to the ARRIVE (Animal Research: Reporting of In Vivo Experiments) guidelines and approved by the Ethical Committee on Animal Experimentation at the School of Dentistry at Araraquara, São Paulo State University UNESP (Protocol Number: 23/2012). Adult Holtzman rats $(n=32)$ of $300 \mathrm{~g}$ were housed in the animal facility of the university and provided standard laboratory food and water ad libitum. The rats were randomly divided into four groups: a) untreated control, b) periodontitis, c) tooth movement, d) combination of periodontitis and tooth movement. Experimental periodontitis was induced by ligatures under anesthesia with intramuscular injections of ketamine chlorhydrate $10 \%(0.08 \mathrm{~mL} / 100 \mathrm{~g}$ body weight) and xylazine chlorhydrate $2 \%$ ( $0.04 \mathrm{~mL} / 100 \mathrm{~g}$ body weight), as previously reported [16-18]. A cotton ligature was tied around the cervical area of the first molars in the maxilla, and the knot was placed mesially. In order to perform orthodontic tooth movement, a closed-coil nickel-titanium spring (Sentalloy®, GAC, Dentsply) providing a relatively constant force of $25 \mathrm{~g}$ was connected between the maxillary first molar and maxillary central incisor teeth, as previously published [16]. To prevent displacement of the $0.20-\mathrm{mm}$ steel wire $(\mathrm{CrNi}, 55.01 .208$, Morelli, Brazil), grooves were prepared on the maxillary central incisor teeth. Afterwards, the wire in the groove was covered with a thin layer of composite resin. For placement of the spring on the maxillary first molars, composite resin was placed on the occlusal surface and, subsequently, the spring was positioned over it. The first molars in the lower jaw were removed to avoid occlusal interferences. Following 5 days of periodontitis induction, orthodontic tooth movement was initiated for 1 day and 3 days. Afterwards, 4 animals per group and time were sacrificed. Subsequently, the gingiva of the 
maxillary first molars was gently harvested for RNA extraction with subsequent real-time PCR.

\section{Human periodontal fibroblasts}

Human periodontal ligament fibroblasts were obtained from periodontally healthy teeth, which became available during wisdom tooth surgery or tooth extraction procedures for orthodontic indications (see below). Approval of the Ethics Committee of the University of Bonn was obtained (\#043/11), and written informed consent was provided by the patients. The periodontal fibroblasts were harvested from the middle third of the root surface and cultured in Dulbecco's minimal essential medium (DMEM, Invitrogen, Karlsruhe, Germany) supplemented with $10 \%$ fetal bovine serum (FBS, Invitrogen), $100-\mathrm{U} / \mathrm{mL}$ penicillin and $100-\mu \mathrm{g} / \mathrm{mL}$ streptomycin (Invitrogen) at $37{ }^{\circ} \mathrm{C}$ in a humidified atmosphere of $5 \% \mathrm{CO}_{2}$. The periodontal fibroblasts were used between $3 \mathrm{rd}$ and 5 th passages. Prior to the experiments, the FBS concentration was reduced to $1 \%$ and the medium was changed every second day during the experiments. To simulate periodontal infection in vitro, cells were exposed to F. nucleatum ATCC 25586 $\left(\mathrm{OD}_{660}: 0.05\right)$. The bacteria were suspended in phosphatebuffered saline $\left(\mathrm{OD}_{660}=1.0\right.$, equivalent to $1.2 \times 10^{9}$ bacterial cells $/ \mathrm{mL}$ ) and subjected twice to ultrasonication $(160 \mathrm{~W}$ for $15 \mathrm{~min})$. Colony-forming units were determined before and after ultrasonication of the suspension. No viable bacteria were found. To mimic orthodontic tooth movement in vitro, periodontal fibroblasts were subjected to constant tensile forces (CTS) by using an established cell strain device for up to 2 days, as previously demonstrated $[19,20]$. Additionally, cells were subjected simultaneously to F. nucleatum and CTS. Untreated cells served as controls.

\section{Scanning electron microscopy photographs}

Scanning electron microscopy was performed at the Center of Electron Microscopy, University Hospital of Jena, Jena (Germany), to see the attachment of $F$. nucleatum on periodontal fibroblasts. Cells were cultured on glass slides, and when a monolayer was formed, cells were exposed to F. nucleatum ATCC 25586 for $1 \mathrm{~h}$. Afterwards, periodontal fibroblasts were fixed in $2 \%$ glutaraldehyde in cacodylate buffer for $30 \mathrm{~min}$, washed twice with cacodylate buffer, and dehydrated using a $10 \%$ graded ethanol series. Critical point drying was performed, and then samples were sputter-coated with gold. Samples were evaluated using a ZEISS LEO-1530 Gemini (Carl Zeiss NTS GmbH, Oberkochen, Germany) equipped with a field emission electron gun at $10 \mathrm{keV}$.

\section{Gene expression of CXCL1, CCL2, and CCL5}

Total RNA extraction was carried out by using the RNeasy Mini Kit (Qiagen, Hilden, Germany) according to manufacturer's protocol. RNA concentration was analyzed by the NanoDrop ND-2000 (Thermo Fisher Scientific, Wilmington, DE, USA) spectrophotometer, and $500 \mathrm{ng}$ of total RNA was reversely transcribed using the iScript ${ }^{\mathrm{TM}}$ Select cDNA Synthesis Kit (Bio-Rad Laboratories, Munich, Germany) at $42{ }^{\circ} \mathrm{C}$ for $90 \mathrm{~min}$ followed by $85^{\circ} \mathrm{C}$ for $5 \mathrm{~min}$ as per manufacturer's instructions. The gene expressions of CXCL1, CCL2, and CCL5 were subsequently determined in triplicate by using QuantiTect Primers (Qiagen), SYBR Green QPCR Master Mix (Bio- Rad), and the iCycler iQ ${ }^{\mathrm{TM}}$ Real-Time PCR Detection System (Bio-Rad). Amplification was performed under the following conditions: initial denaturation at $95{ }^{\circ} \mathrm{C}$ for $5 \mathrm{~min}$, followed by 40 cycles of denaturation at $95^{\circ} \mathrm{C}$ for $10 \mathrm{~s}$, and combined annealing/extension at $60{ }^{\circ} \mathrm{C}$ for $30 \mathrm{~s}$. Glyceraldehyde-3- phosphate dehydrogenase was used as a housekeeping gene, and all data were analyzed by using the comparative threshold cycle method.

\section{Protein levels of CXCL1, CCL2, and CCL5}

Human gingival biopsies were fixed in 4\% paraformaldehyde (Sigma-Aldrich) for 2 days, dehydrated in an ascending ethanol series (AppliChem, Darmstadt, Germany), embedded in paraffin (McCormick Scientific, Richmond, IL, USA), sectioned at $2.5 \mu \mathrm{m}$ thickness, mounted onto glass slides (Carl Roth, Karlsruhe, Germany), and dried at $37{ }^{\circ} \mathrm{C}$ overnight. Following deparaffinization and rehydration, the sections were rinsed in PBS for $2 \mathrm{~min}$. Afterwards, the endogenous peroxidase was blocked by using $0.3 \%$ methanol (AppliChem) $/ \mathrm{H}_{2} \mathrm{O}_{2}$ (Merck Eurolab) solution for $5 \mathrm{~min}$. The sections were then blocked with goat serum (Dako) for $20 \mathrm{~min}$ and incubated with rabbit anti-CXCL1, anti-CCL2, or antiCCL5 polyclonal antibody (all Abcam; 1:100) in a humid chamber at $4{ }^{\circ} \mathrm{C}$ overnight. After washing, the sections were incubated with goat anti-rabbit IgG-HRP secondary antibody (Dako) at room temperature for $30 \mathrm{~min}$. The peroxidase activity was visualized with 3.3 '-diaminobenzidine chromogen (Thermo Fisher Scientific). Finally, slides were rinsed and counterstained with Mayer's hematoxylin (Merck Eurolab) for $1 \mathrm{~min}$. The images were taken with an Axioskop 2 microscope and, subsequently, analyzed with the AxioVision 4.7 software.

Protein levels of CXCL1, CCL2, and CCL5 in supernatants of periodontal fibroblasts at 1 day and 2 days were measured with a commercially available enzyme-linked immunosorbent assay (ELISA) kit (LSBio, Seattle, WA, USA) according to the manufacturer's protocol. Final absorbance was analyzed with a microplate reader (PowerWave x, BioTek Instruments, Winooski, VT, USA) at $450 \mathrm{~nm}$. For data normalization, the 
periodontal fibroblasts were detached after collection of the supernatants and counted with an automatic cell counter (Moelab, Hilden, Germany).

\section{Statistical analysis}

For statistical analysis, the IBM SPSS Statistics software (Version 22, IBM SPSS, Chicago, IL, USA) was applied. Mean values and standard errors of the mean (SEM) were calculated for quantitative data. In order to test for significant differences between the groups, the $t$ test, Mann-Whitney $U$ test, and ANOVA followed by the post-hoc Tukey multiple comparison tests were applied. Differences between groups were considered significant at $p<0.05$.

\section{Results}

\section{Presence of CXCL1, CCL2, and CCL5 in human gingival biopsies}

First, we sought to determine if CXCL1, CCL2, and CCL5 are expressed in human gingival biopsies. As evidenced by realtime PCR, significantly $(p<0.05)$ higher expression levels of CXCL1, CCL2, and CCL5 were found in the gingiva from sites of periodontitis as compared with biopsies from periodontally healthy sites (Fig. 1a-c). These findings at transcriptional level were confirmed by immunohistochemistry analysis, which demonstrated a pronounced immunostaining for CXCL1, CCL2, and CCL5 proteins in the gingiva of periodontitis specimens (Fig. 1d-f). Interestingly, the chemokines were mainly present in the subepithelial region. By contrast, clinically healthy gingiva did not show a marked presence of these chemokines (Fig. 1d-f).

\section{Presence of CXCL1, CCL2, and CCL5 in gingival biopsies of rats with experimental periodontitis and/or orthodontic tooth movement}

Since the aforementioned analyses revealed that the levels of all chemokines were increased at sites of periodontitis, we wondered if biomechanical forces could regulate the bacteria-induced chemokine expressions. To study the interactions of microbial and biomechanical signals in a controlled environment, an animal model was used. The rats were subjected to experimental periodontitis and/or orthodontic tooth movement, untreated rats served as control. As shown in Fig. $2 \mathrm{a}-\mathrm{c}$, periodontitis resulted in significantly $(p<0.05)$ enhanced expression levels of CXCL1, CCL2, and CCL5 in the gingival biopsies. However, when the sites of experimental periodontitis were also subjected to orthodontic tooth movement, the gingival levels of all chemokines were significantly $(p<0.05)$ lower as compared with the periodontitis group, demonstrating that the orthodontic forces counteracted the bacteria-induced upregulation of CXCL1, CCL2, and CCL5 (Fig. 2a-c).

\section{Regulation of CXCL1, CCL2, and CCL5 by F. nucleatum and/or biomechanical forces in fibroblasts}

Since $F$. nucleatum is a pathogen which plays a critical role in both gingivitis and periodontitis, periodontal fibroblasts were exposed to this bacterium in further in vitro experiments (Fig. 3). As depicted in Fig. 4a, F. nucleatum caused a significant $(p<0.05)$ upregulation of CXCL1 in the cells at 1 day, as analyzed by real-time PCR. However, when these cells were subjected simultaneously to F. nucleatum and CTS, the $F$. nucleatum-induced upregulation of CXCL1 was significantly $(p<0.05)$ inhibited (Fig. 4a). Like CXCL1, CCL2 and CCL5 were significantly $(p<0.05)$ increased in the presence of F. nucleatum (Fig. 4 b and c). Furthermore, the upregulated CCL2 and CCL5 expressions were significantly $(p<0.05)$ diminished again, when the fibroblasts were treated with a combination of F. nucleatum and CTS (Fig. $4 \mathrm{~b}$ and c). As evidenced by ELISA, exposure of the cells with $F$. nucleatum also resulted in significantly $(p<0.05)$ enhanced protein levels of CXCL1, CCL2, and CCL5. Moreover, application of CTS to the cells counteracted the stimulatory action of F. nucleatum on CXCL1, CCL2, and CCL5 proteins, confirming and expanding the findings at transcriptional level (Fig. 4d-f).

\section{Discussion}

This study provides original evidence in vitro and in vivo that the chemokines CXCL1, CCL2, and CCL5 are regulated by both microbial and biomechanical signals in periodontal cells and tissues. Furthermore, our study revealed that biomechanical forces can counteract the stimulatory actions of F. nucleatum on these chemokines. Therefore, biomechanical loading might aggravate periodontal infection by compromising the recruitment of immunoinflammatory cells.

Periodontitis is characterized by a chronic inflammation caused by subgingival microorganisms [2]. The aim of the inflammatory host response is to eliminate or at least to reduce the pathogenic bacteria, thereby protecting the periodontal cells, tissues, and structure. In order to recruit immunoinflammatory cells to the site of the microbial attack and tissue destruction, chemokines, such as CXCL1, CCL2, and CCL5, are required. Whereas CXCL1 is a strong chemoattractant for neutrophils, CCL2 and CCL5 mainly promote the recruitment of monocytes, macrophages, and lymphocytes [13]. Several studies have clearly demonstrated that these cell types and their subsets have defined roles in the battle between the host and microorganisms in periodontitis [21,22]. 


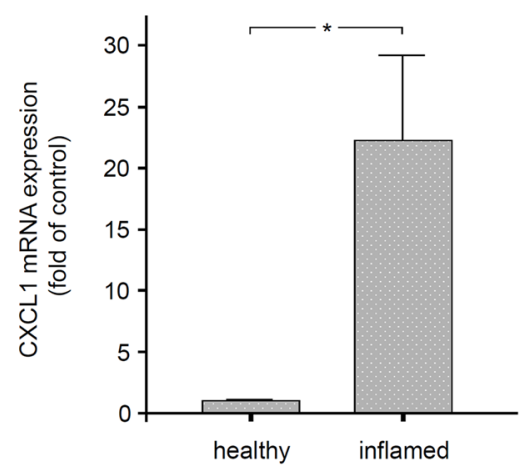

CXCL1

d
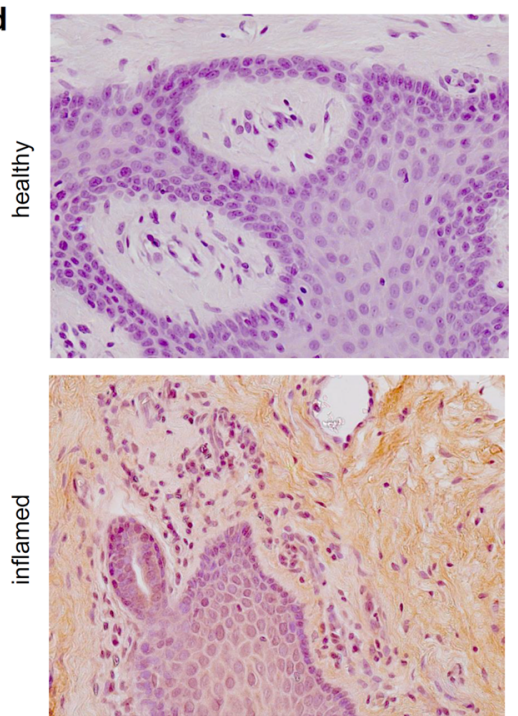

b

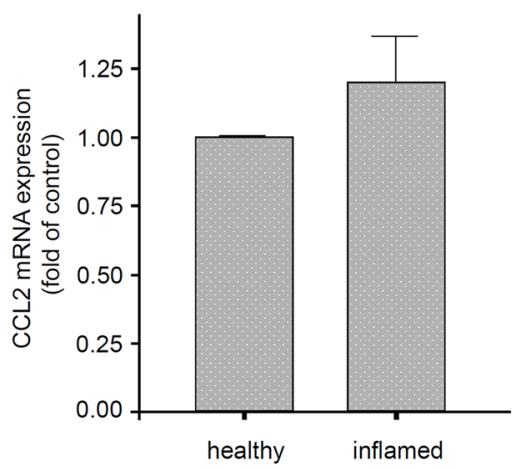

CCL2

e

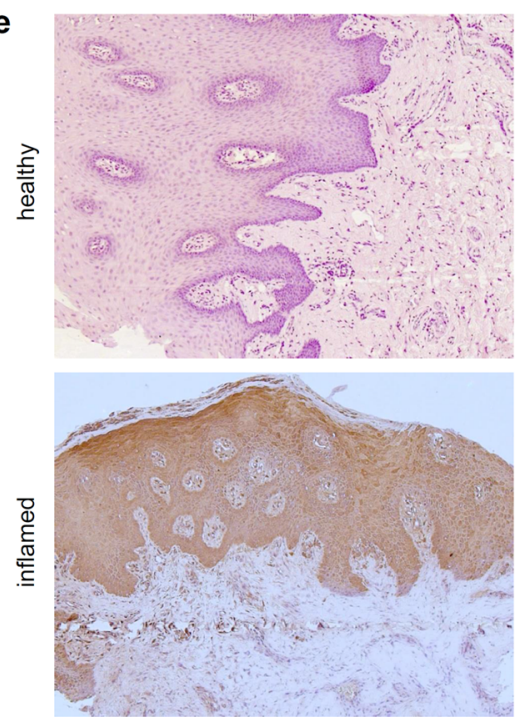

C

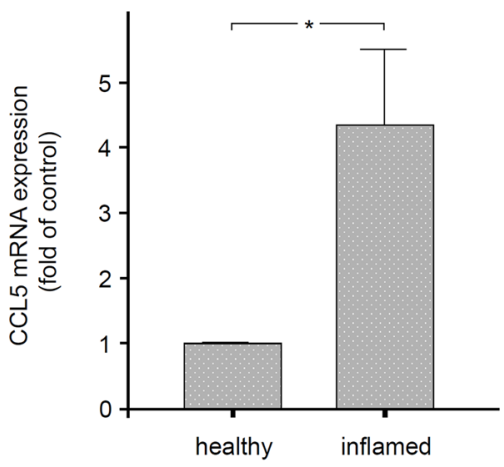

CCL5

f
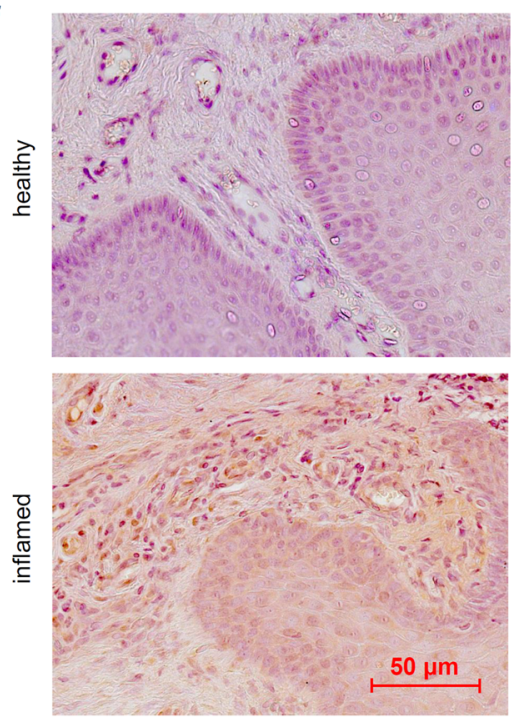

Fig. 1 CXCL1, CCL2, and CCL5 in human gingival biopsies. Gene expression of CXCL1 (a), CCL2 (b), and CCL5 (c) in human gingival biopsies from sites of periodontitis $(n=6)$ as compared with periodontally healthy sites $(n=6)$, as analyzed by real-time PCR. Mean values and SEM, *significant $(p<0.05)$ difference between groups. CXCL1 (d),

Our study proves that CXCL1, CCL2, and CCL5 are of utmost importance in the pathogenesis of periodontal diseases.
CCL2 (e), and CCL5 (f) proteins in human gingival biopsies from periodontally healthy and inflamed sites, as analyzed by immunohistochemistry. Representative histological sections from a periodontally healthy subject and a periodontitis patient are shown

They were upregulated in human gingiva from sites of periodontitis, in inflamed gingiva from rats and also in periodontal

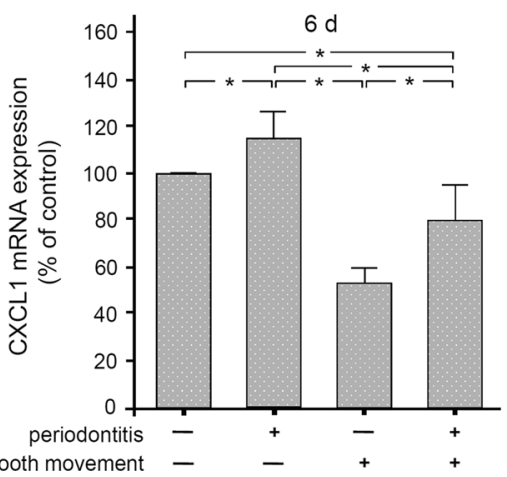

b

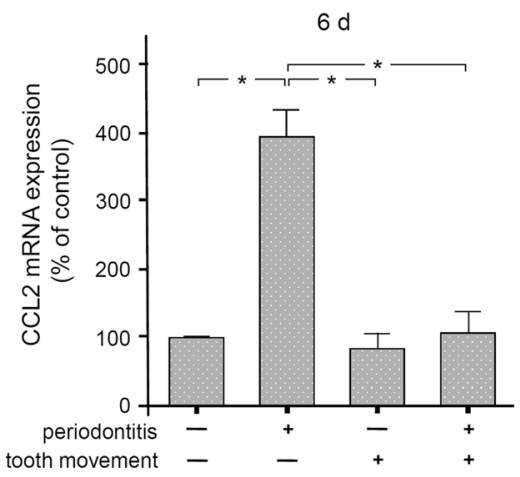

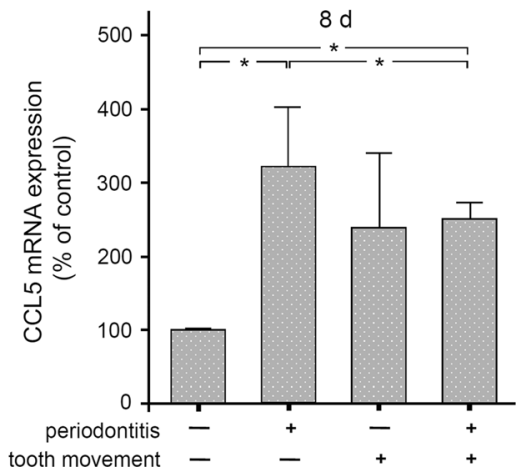

Fig. 2 CXCL1, CCL2, and CCL5 in rat gingival biopsies. Gene expression of CXCL1 (a), CCL2 (b), and CCL5 (c) in gingival biopsies of rats subjected to experimental periodontitis and/or orthodontic tooth movement, as analyzed by real-time PCR. Untreated rats served as control. Mean values and SEM ( $n=4$ animals/group), *significant $(p<0.05)$ difference between groups 

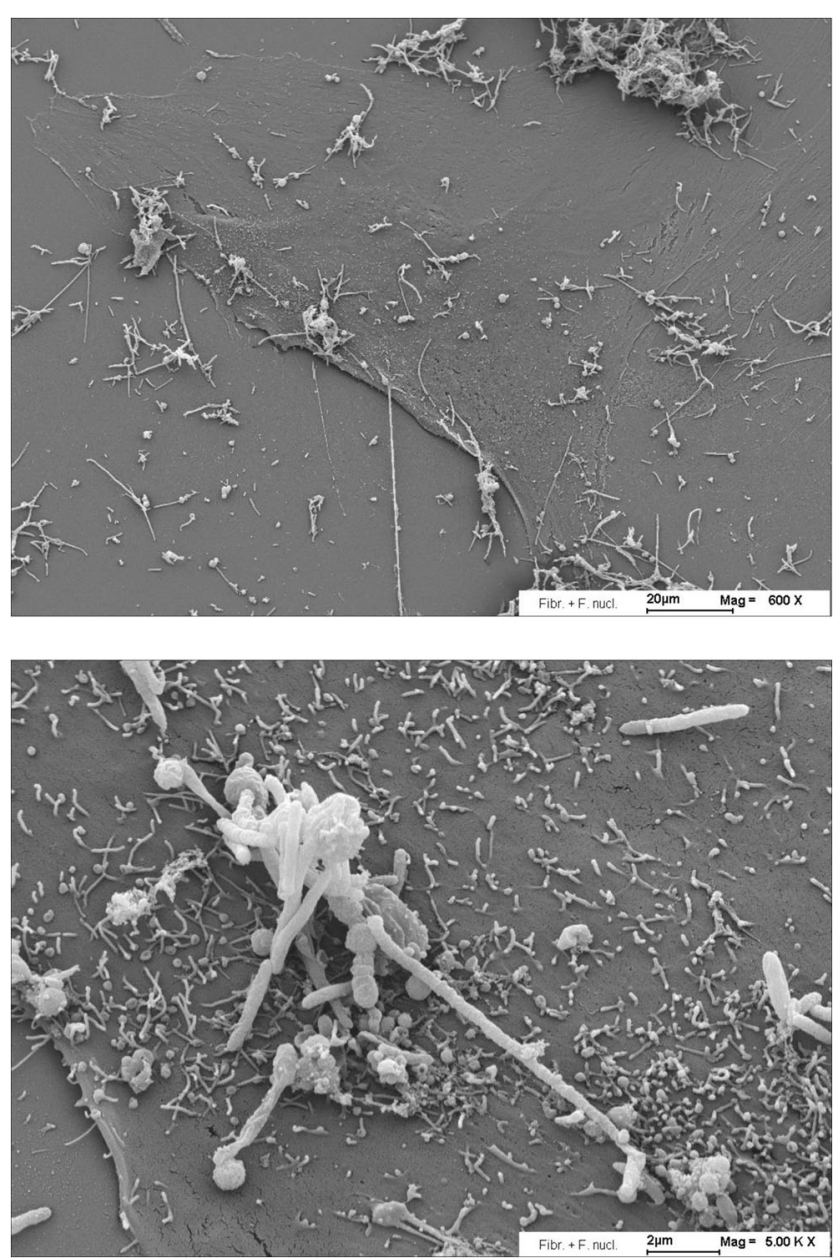

Fig. 3 F. nucleatum and periodontal fibroblast, as depicted by scanning electron microscopy

fibroblasts exposed to the periodontopathogen $F$. nucleatum. These transcriptional results were further supported by findings at protein level, as evidenced by immunohistochemistry and ELISA. Interestingly, the role of the aforementioned chemokines has also been addressed by other investigators. For example, increased CXCL1, CCL2, and CCL5 levels have been found in gingival crevicular fluid (GCF) from periodontally diseased sites as compared with healthy sites [10, 23, 24]. Moreover, elevated CCL2 levels have been observed in human gingival tissues affected by periodontitis $[25,26]$. These observations concur with our findings, indicating that periodontal cells and tissues produce enhanced levels of CXCL1, CCL2, and CCL5 in the presence of periodontal infection and may therefore contribute to elevated levels of these chemokines in GCF. Notably, increased serum levels of CXCL1 and CCL5 have been demonstrated in experimental periodontitis in mice $[6,27]$. Whether the locally produced levels of CXCL1, CCL2, and CCL5 from the periodontal tissues contribute to the elevated serum levels of these chemokines should be further analyzed.
Few studies have also focused on the actions of mechanical forces on the chemokine production in periodontal cells and tissues. Periodontal cells subjected to compressive forces for $24 \mathrm{~h}$ produced higher CCL2 levels as controls [28]. Increased CCL2 periodontal tissue levels following mechanical force application were also observed in animal models [28, 29]. These findings are in contrast to our results, which could be due to different reasons. For example, their in vivo studies focused on CCL2 in periodontal ligament of the compression site, whereas we examined CCL2 in gingiva without restriction to a particular site. In vitro, we subjected periodontal ligament cells to tensile strain in contrast to compressive forces. In humans, the effect of mechanical forces on CCL2 and CCL 5 has been studied during orthodontic tooth movement and yielded controversial results [30-32]. The regulation of these chemokines may depend on several parameters, such as duration, magnitude, and type of force application as well as the biological material evaluated.

In our study, the bacteria-induced upregulation of CXCL1, CCL2, and CCL5 was surprisingly counteracted by biomechanical forces in vitro and in vivo, suggesting that biomechanical loading may compromise a sufficient recruitment of immunoinflammatory cells in periodontitis. This mechanism may explain how occlusal overloading may aggravate periodontitis, as it has clearly been shown in animals [33]. Interestingly, CXCL1 and CCL5 promote migration of gingival fibroblasts and CCL5 stimulates proliferation of these cells [34], indicating that these chemokines affect not only neutrophils, monocytes/macrophages, and lymphocytes but also periodontal cells.

Although it was easy to obtain human gingival biopsies from sites of periodontitis and periodontal health, gingival tissues from sites affected by both periodontitis and orthodontic forces become hardly available. Therefore, an animal model was chosen to study the interaction of periodontitis and biomechanical/orthodontic forces. In this model, periodontitis was induced by cotton ligatures around the maxillary first molars, which promoted the accumulation of a complex bacterial biofilm and bone resorption, as previously shown [16]. Extrapolating data from rats to humans has to be done with caution. However, a study by Hyde et al. on the characterization of the rat oral microbiome has demonstrated that there are sufficient similarities in oral microbiome structure and physiological effects to justify the use of rat models, even if there are limitations [35]. We also wanted to study the interactions between microbial and mechanical signals at cellular levels and therefore exposed periodontal fibroblast to both F. nucleatum and CTS, which was applied by the use of an established cell strain device. However, during orthodontic tooth movement and mastication, periodontal tissues are subjected to complex mechanical loading. Whether compressive, hydrostatic, and shear forces as well as their combinations exert similar regulatory effects on CXCL1, CCL2, and 


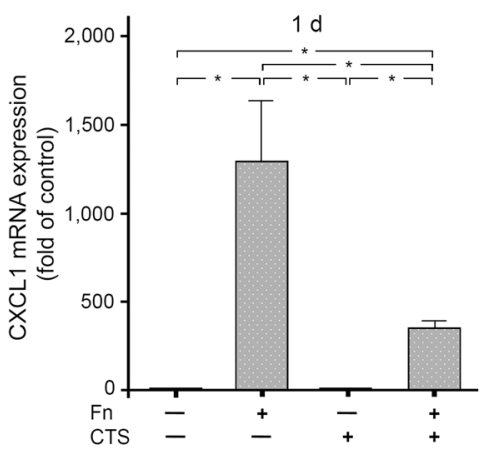

b

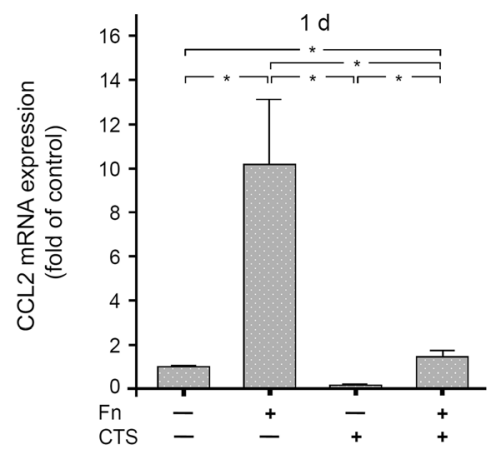

C

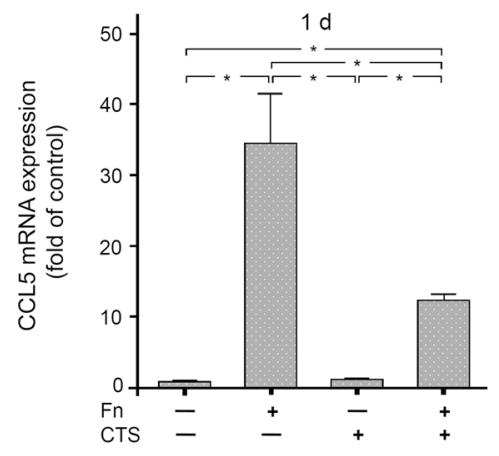

d

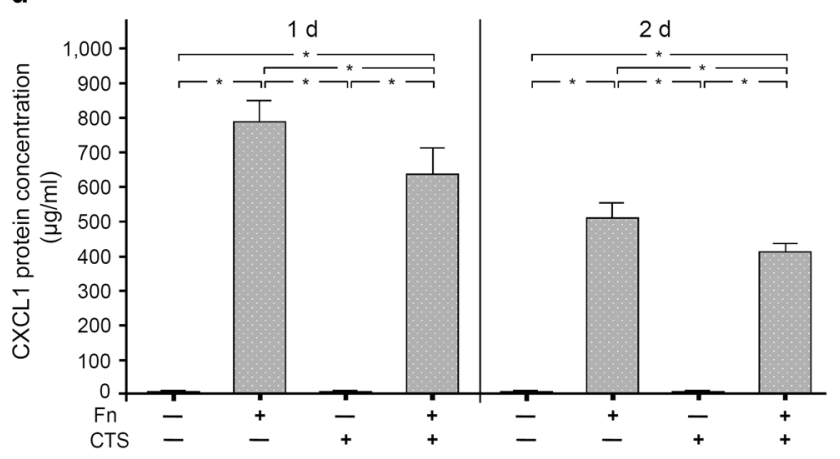

e

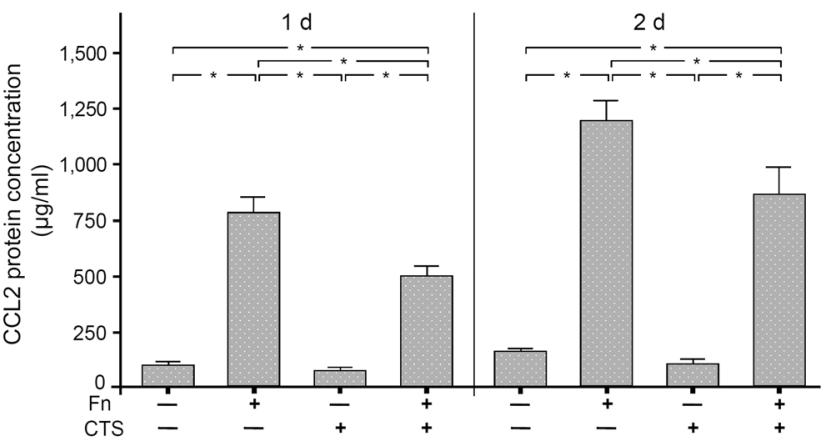

f

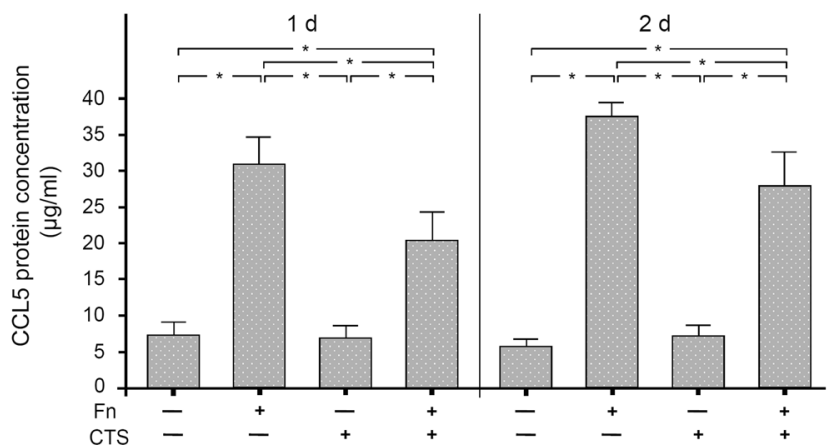

Fig. 4 CXCL1, CCL2, and CCL5 in human periodontal fibroblasts. Gene expression of CXCL1 (a), CCL2 (b), and CCL5 (c) in human periodontal fibroblasts exposed to F. nucleatum and/or constant tensile strain (CTS) at 1 day, as analyzed by real-time PCR. Protein levels of CXCL1 (d), CCL2 (e), and CCL5 (f) in supernatants of human periodontal fibroblasts exposed to $F$. nucleatum and/or constant tensile strain (CTS) at 1 day and 2 days, as analyzed by ELISA. Mean values and SEM ( $n=9 /$ group), *significant $(p<0.05)$ difference between groups

single bacterium but also is a highly complex biofilm [37]. Future studies should therefore focus on the regulatory actions of other pathogenic microorganisms and their combinations on CXCL1, CCL2, and CCL5.

Since $F$. nucleatum is strongly involved in periodontal diseases, this microorganism was used to simulate periodontal infection in our in vitro study. Nevertheless, Porphyromonas gingivalis is another important and well-studied periodontopathogen. Intriguingly, $P$. gingivalis and Escherichia coli have also been shown to enhance CXCL1, 
CCL2, and CCL5 expressions in human periodontal cells [38-42]. By demonstrating that these chemokines are upregulated in response to microbial stimuli, these studies support our in vitro findings. In our experiments, a suspension of F. nucleatum, which was exposed to intensive ultrasonication before application, was used. Therefore, it can be assumed that the suspension mainly contained disrupted cell wall particles with a high amount of lipopolysaccharides.

Future studies should also unravel the interactions between the microbial and mechanical signals at receptor level and within the cells. In addition, it would be intriguing to know if microbial and mechanical signals also interact on the regulation of chemokine receptors in periodontal cells and tissues. Moreover, periodontal cells with a fibroblast-like phenotype were used in our in vitro experiments. However, if other periodontal cell phenotypes, such as osteoblasts and cementoblasts, exert similar effects on CXCL1, CCL2, and CCL5 remains to be studied.

\section{Conclusions}

In summary, our in vitro and in vivo investigations have shown that the chemokines CXCL1, CCL2, and CCL5 are regulated by both bacterial and mechanical signals in periodontal cells and tissues. Additionally, our study revealed that biomechanical forces can inhibit the stimulatory actions of F. nucleatum on CXCL1, CCL2, and CCL5. Therefore, biomechanical loading may aggravate periodontal infection by compromising the critical recruitment of immunoinflammatory cells.

Acknowledgments The authors would like to thank Mrs. Ramona Menden, Mrs. Silke van Dyck, Mrs. Jana Marciniak, Prof. Sören Jepsen, and Prof. Gerhard Wahl for their valuable support.

Authors' contributions Defining the study aims: Birgit Rath-Deschner and Andressa V. B. Nogueira; coordination of collaboration: Andreas Jäger and James Deschner; planning the experiments: Birgit RathDeschner, Andressa V. B. Nogueira, Joni A. Cirelli, Anna Damanaki, James Deschner, Marjan Nokhbehsaim, and Svenja Memmert; growing the bacteria and preparing the bacterial lysate: Sigrun Eick; performing the experiments: Birgit Rath-Deschner, Andressa V. B. Nogueira, Anna Damanaki, Marjan Nokhbehsaim, and Svenja Memmert; monitoring/ supervising the experiments: Andreas Jäger, James Deschner, and Joni A. Cirelli; analyzing and discussing the data: Birgit Rath-Deschner, Andressa V. B. Nogueira, Anna Damanaki, Svenja Memmert, James Deschner, Werner Götz, and Andreas Jäger; creating the figures: Birgit Rath-Deschner and Andressa V. B. Nogueira. All authors contributed to the writing of the manuscript. All authors read and approved the final manuscript.

Funding information Open Access funding provided by Projekt DEAL. This study was supported by the German Society of Orthodontics (DGKFO), the São Paulo Research Foundation, Brazil (FAPESP: 2014/20715-7, 2017/07137-2) and the German Research Foundation (DE1593/5-1), Coordenação de Aperfeiçoamento de Pessoal de Nível
Superior, CAPES, Brazil within the Program Capes/Probral (ID 88881.144012/2017-01) and German Academic Exchange Service, DAAD, Germany (ID 57391253).

\section{Compliance with ethical standards}

Conflict of interest The authors declare that they have no conflict of interest.

Ethical approval All procedures performed in studies involving human participants were in accordance with the ethical standards of the Ethics Committee of the University of Bonn (043/11) and with the 1964 Helsinki declaration and its later amendments or comparable ethical standards.

All procedures performed in this study were in accordance with the ethical standards of the Ethical Committee for Animal Use (CEUA) of the School of Dentistry at Araraquara - UNESP (23/2012) and performed in accordance with the guideline from the Brazilian College for Animal Experimentation (COBEA). All applicable international, national, and/ or institutional guidelines for the care and use of animals were followed.

Informed consent Informed consent was obtained from all individual participants included in the study.

Open Access This article is licensed under a Creative Commons Attribution 4.0 International License, which permits use, sharing, adaptation, distribution and reproduction in any medium or format, as long as you give appropriate credit to the original author(s) and the source, provide a link to the Creative Commons licence, and indicate if changes were made. The images or other third party material in this article are included in the article's Creative Commons licence, unless indicated otherwise in a credit line to the material. If material is not included in the article's Creative Commons licence and your intended use is not permitted by statutory regulation or exceeds the permitted use, you will need to obtain permission directly from the copyright holder. To view a copy of this licence, visit http://creativecommons.org/licenses/by/4.0/.

\section{References}

1. Slots J (2017) Periodontitis: facts, fallacies and the future. Periodontol 2000(75):7-23. https://doi.org/10.1111/prd.12221

2. Meyle J, Chapple I (2015) Molecular aspects of the pathogenesis of periodontitis. Periodontol 2000 69:7-17. https://doi.org/10.1111/ prd. 12104

3. He J, Huang W, Pan Z, Cui H, Qi G, Zhou X, Chen H (2012) Quantitative analysis of microbiota in saliva, supragingival, and subgingival plaque of Chinese adults with chronic periodontitis. Clin Oral Investig 16:1579-1588. https://doi.org/10.1007/s00784011-0654-4

4. Signat B, Roques C, Poulet P, Duffaut D (2011) Fusobacterium nucleatum in periodontal health and disease. Curr Issues Mol Biol 13:25-36

5. Rossi D, Zlotnik A (2000) The biology of chemokines and their receptors. Annu Rev Immunol 18:217-242. https://doi.org/10. 1146/annurev.immunol.18.1.217

6. Scanu A, Giraudo C, Galuppini F, Lazzarin V, Pennelli G, Sivolella S, Stellini E, Oliviero F, Galozzi P, Rugge M, Stramare R, Luisetto R, Punzi L (2019) Periodontal injection of lipopolysaccharide promotes arthritis development in mice. Inflammation 42:1117-1128. https://doi.org/10.1007/s10753-019-00975-6 
7. Ren G, Lutz I, Railton P, Wiley JP, McAllister J, Powell J, Krawetz RJ (2018) Serum and synovial fluid cytokine profiling in hip osteoarthritis: distinct from knee osteoarthritis and correlated with pain. BMC Musculoskelet Disord 19:39. https://doi.org/10.1186/s12891018-1955-4

8. Gupta M, Chaturvedi R, Jain A (2013) Role of monocyte chemoattractant protein-1 (MCP-1) as an immune-diagnostic biomarker in the pathogenesis of chronic periodontal disease. Cytokine 61:892-897. https://doi.org/10.1016/j.cyto.2012.12.012

9. Maury E, Brichard SM, Pataky Z, Carpentier A, Golay A, Bobbioni-Harsch E (2010) Effect of obesity on growth-related oncogene factor-alpha, thrombopoietin, and tissue inhibitor metalloproteinase-1 serum levels. Obesity (Silver Spring) 18: 1503-1509. https://doi.org/10.1038/oby.2009.464

10. Kurtis B, Tuter G, Serdar M, Akdemir P, Uygur C, Firatli E, Bal B (2005) Gingival crevicular fluid levels of monocyte chemoattractant protein-1 and tumor necrosis factor-alpha in patients with chronic and aggressive periodontitis. J Periodontol 76: 1849-1855. https://doi.org/10.1902/jop.2005.76.11.1849

11. Reape TJ, Groot PHE (1999) Chemokines and atherosclerosis. Atherosclerosis 147:213-225. https://doi.org/10.1007/s11883001-0067-y

12. Rajarathnam K, Schnoor M, Richardson RM, Rajagopal S (2019) How do chemokines navigate neutrophils to the target site: dissecting the structural mechanisms and signaling pathways. Cell Signal 54:69-80. https://doi.org/10.1016/j.cellsig.2018.11.004

13. Atri C, Guerfali FZ, Laouini D (2018) Role of human macrophage polarization in inflammation during infectious diseases. Int J Mol Sci 19:1801. https://doi.org/10.3390/ijms19061801

14. Feller L, Khammissa RA, Schechter I, Thomadakis G, Fourie J, Lemmer J (2015) Biological events in periodontal ligament and alveolar bone associated with application of orthodontic forces. ScientificWorldJournal 2015:876509. https://doi.org/10.1155/ 2015/876509

15. Krishnan V, Davidovitch Z (2009) On a path to unfolding the biological mechanisms of orthodontic tooth movement. J Dent Res 88: 597-608. https://doi.org/10.1177/0022034509338914

16. Nogueira AV, de Molon RS, Nokhbehsaim M, Deschner J, Cirelli JA (2017) Contribution of biomechanical forces to inflammationinduced bone resorption. J Clin Periodontol 44:31-41. https://doi. org $/ 10.1111 /$ jcpe. 12636

17. Nogueira AV, de Souza JA, de Molon RS, Pereira Eda S, de Aquino SG, Giannobile WV, Cirelli JA (2014) HMGB1 localization during experimental periodontitis. Mediat Inflamm 2014:816320. https:// doi.org/10.1155/2014/816320

18. de Souza JA, Nogueira AV, de Souza PP, Cirelli JA, Garlet GP, Rossa C Jr (2011) Expression of suppressor of cytokine signaling 1 and 3 in ligature-induced periodontitis in rats. Arch Oral Biol 56: 1120-1128. https://doi.org/10.1016/j.archoralbio.2011.03.022

19. Deschner J, Rath-Deschner B, Reimann S, Bourauel C, Götz W, Jepsen S, Jäger A (2007) Regulatory effects of biophysical strain on rat TMJ discs. Ann Anat 189:326-328. https://doi.org/10.1016/j. aanat.2007.02.004

20. Rath-Deschner B, Deschner J, Reimann S, Jager A, Götz W (2009) Regulatory effects of biomechanical strain on the insulin-like growth factor system in human periodontal cells. J Biomech 42: 2584-2589. https://doi.org/10.1016/j.jbiomech.2009.07.013

21. Sima C, Viniegra A, Glogauer M (2019) Macrophage immunomodulation in chronic osteolytic diseases-the case of periodontitis. J Leukoc Biol 105:473-487. https://doi.org/10.1002/ JLB.1RU0818-310R

22. Alvarez C, Rojas C, Rojas L, Cafferata EA, Monasterio G, Vernal R (2018) Regulatory T lymphocytes in periodontitis: a translational view. Mediat Inflamm 2018:7806912. https://doi.org/10.1155/ 2018/7806912
23. Sakai A, Ohshima M, Sugano N, Otsuka K, Ito K (2006) Profiling the cytokines in gingival crevicular fluid using a cytokine antibody array. J Periodontol 77:856-864. https://doi.org/10.1902/jop.2006. 050340

24. Gamonal J, Acevedo A, Bascones A, Jorge O, Silva A (2000) Levels of interleukin-1 beta, -8 , and -10 and RANTES in gingival crevicular fluid and cell populations in adult periodontitis patients and the effect of periodontal treatment. J Periodontol 71:15351545. https://doi.org/10.1902/jop.2000.71.10.1535

25. Boström EA, Kindstedt E, Sulniute R, Palmqvist P, Majster M, Holm CK, Zwicker S, Clark R, Önell S, Johansson I, Lerner UH, Lundberg P (2015) Increased eotaxin and MCP-1 levels in serum from individuals with periodontitis and in human gingival fibroblasts exposed to pro-inflammatory cytokines. PLoS One 10: e0134608. https://doi.org/10.1371/journal.pone.0134608

26. Hanazawa S, Kawata Y, Takeshita A et al (1993) Expression of monocyte chemoattractant protein 1 (MCP-1) in adult periodontal disease: increased monocyte chemotactic activity in crevicular fluids and induction of MCP-1 expression in gingival tissues. Infect Immun 61:5219-5224

27. Palioto DB, Finoti LS, Kinane DF, Benakanakere M (2019) Epigenetic and inflammatory events in experimental periodontitis following systemic microbial challenge. J Clin Periodontol. https:// doi.org/10.1111/jcpe.13151

28. Asano M, Yamaguchi M, Nakajima R, Fujita S, Utsunomiya T, Yamamoto H, Kasai K (2011) IL-8 and MCP-1 induced by excessive orthodontic force mediates odontoclastogenesis in periodontal tissues. Oral Dis 17:489-498. https://doi.org/10.1111/j.1601-0825. 2010.01780.x

29. Alhashimi N, Frithiof L, Brudvik P, Bakhiet M (1999) Chemokines are upregulated during orthodontic tooth movement. J Interf Cytokine Res 19:1047-1052. https://doi.org/10.1089/ 107999099313271

30. Madureira DF, Taddei Sde A, Abreu MH, Pretti H, Lages EM, da Silva TA (2012) Kinetics of interleukin-6 and chemokine ligands 2 and 3 expression of periodontal tissues during orthodontic tooth movement. Am J Orthod Dentofac Orthop 142:494-500. https:// doi.org/10.1016/j.ajodo.2012.05.012

31. Capelli J Jr, Kantarci A, Haffajee A, Teles RP, Fidel R Jr, Figueredo CM (2011) Matrix metalloproteinases and chemokines in the gingival crevicular fluid during orthodontic tooth movement. Eur J Orthod 33:705-711. https://doi.org/10.1093/ejo/cjq148

32. Garlet TP, Coelho U, Repeke CE, Silva JS, Cunha Fde Q, Garlet GP (2008) Differential expression of osteoblast and osteoclast chemmoatractants in compression and tension sides during orthodontic movement. Cytokine 42:330-335. https://doi.org/10.1016/j. cyto.2008.03.003

33. Junqueira RB, Saavedra GSFA, Macedo NL Considerations about the relation between occlusal trauma and periodontal/peri-implant disease. Braz Dent Sci 18:9-14. https://doi.org/10.14295/bds.2015. v18i2.1079

34. Buskermolen JK, Roffel S, Gibbs S (2017) Stimulation of oral fibroblast chemokine receptors identifies CCR3 and CCR4 as potential wound healing targets. J Cell Physiol 232:2996-3005. https://doi.org/10.1002/jcp.25946

35. Hyde ER, Luk B, Cron S, Kusic L, McCue T, Bauch T, Kaplan H, Tribble G, Petrosino JF, Bryan NS (2014) Characterization of the rat oral microbiome and the effects of dietary nitrate. Free Radic Biol Med 77:249-257. https://doi.org/10.1016/j.freeradbiomed.2014. 09.017

36. Saito A, Inagaki S, Kimizuka R, Okuda K, Hosaka Y, Nakagawa T, Ishihara K (2008) Fusobacterium nucleatum enhances invasion of human gingival epithelial and aortic endothelial cells by Porphyromonas gingivalis. FEMS Immunol Med Microbiol 54: 349-355. https://doi.org/10.1111/j.1574-695X.2008.00481.x 
37. Hajishengallis G (2015) Periodontitis: from microbial immune subversion to systemic inflammation. Nat Rev Immunol 15:30-44. https://doi.org/10.1038/nri3785

38. Fitzsimmons TR, Ge S, Bartold PM (2018) Compromised inflammatory cytokine response to P. gingivalis LPS by fibroblasts from inflamed human gingiva. Clin Oral Investig 22:919-927. https:// doi.org/10.1007/s00784-017-2171-6

39. Svensson D, Aidoukovitch A, Anders E, Jönsson D, Nebel D, Nilsson BO (2017) Secretory leukocyte protease inhibitor regulates human periodontal ligament cell production of pro-inflammatory cytokines. Inflamm Res 66:823-831. https://doi.org/10.1007/ s00011-017-1062-2

40. Basso FG, Soares DG, Pansani TN, Turrioni AP, Scheffel DL, de Souza Costa CA, Hebling J (2015) Effect of LPS treatment on the viability and chemokine synthesis by epithelial cells and gingival fibroblasts. Arch Oral Biol 60:1117-1121. https://doi.org/10.1016/ j.archoralbio.2015.04.010

41. Doyle CJ, Fitzsimmons TR, Marchant C, Dharmapatni AA, Hirsch R, Bartold PM (2015) Azithromycin suppresses P. gingivalis LPSinduced pro-inflammatory cytokine and chemokine production by human gingival fibroblasts in vitro. Clin Oral Investig 19:221-227. https://doi.org/10.1007/s00784-014-1249-7

42. Nebel D, Svensson D, Arosenius K, Larsson E, Jönsson D, Nilsson BO (2015) 1 $\alpha, 25$-dihydroxyvitamin D3 promotes osteogenic activity and downregulates proinflammatory cytokine expression in human periodontal ligament cells. J Periodontal Res 50:666-673. https://doi.org/10.1111/jre.12249

Publisher's note Springer Nature remains neutral with regard to jurisdictional claims in published maps and institutional affiliations. 